\title{
Three in one: The model of Sun-Earth-Moon
}

\author{
İbrahim Ünal ${ }^{1 \mathrm{a}}$ and İlda Özdemir ${ }^{2}$ \\ ${ }^{1}$ İnönü University, Faculty of Education, Science Education Department, Malatya, 44280, TURKEY \\ ${ }^{2}$ Ahi Evran University, Faculty of Education, Science Education Department, Kırşehir, 40100, \\ TURKEY
}

\begin{abstract}
The purpose of this study is to develop a Sun-Earth-Moon model which will be used in describing fundamental topics of astronomy, hardly understood correctly because of their three dimensional and complicated nature. Abstract and complex frame of science is the most significant obstacle against comprehension of scientific topics. Students have difficulties in explaining the meaning of the events that they cannot see, hear and touch, and they cannot relate the events and the information they have. So concepts must be supported with material for quality in science education. In this study, a model has been developed to make it easier to describe the real and the apparent motions of the Sun-Earth-Moon system and the results of these motions, a topic within the scope of Astronomy, which has been taught to senior undergraduate students of Science Education Programs, followed in the Faculty of Education in İnönü University. Our Sun-Earth-Moon model is a more complicated and detailed model than previous ones in terms of explaining single setting topics such as eclipses, seasons, night-day cycle and moon phases and in addition, having many features such as orbital and axial inclinations, axial rotation and orbital motion at the same time. As a result of a literature review, this study has showed that the previous models aren't as comprehensive and well-equipped as our model in terms of the features in ours.
\end{abstract}

Keywords. The model of Sun-Earth-Moon, astronomy education, model development.

\section{Introduction}

Abstract and complex frame of science has made models indispensable in teaching science. Students have difficulties in explaining the meaning of the events that they cannot see, hear and touch and can't relate the events and the information they have. If these abstract events are taught with graphics, symbols, pictures or three dimensional models, students will have

\footnotetext{
a Corresponding author: ibrahim.unal@inonu.edu.tr
} 
an opportunity to observe and review the case, which will turn into being concrete from being abstract $[6,10,4,2,14,8,12]$.

Abstract and complex frame of science is the the most significant obstacle against comprehension of scientific topics. Students usually don't understand what they don't observe or examine. We think that even if a student is able to imagine an event, his/her imagination can be misleading because he/she will not be using his/her senses, which could cause misconceptions, very hard to compensate for. We think that we are able to teach the structure of an atom to students. While we are talking about concepts such as nuclei, protons, electrons and orbits in the class, it is possible to encounter with vague facial expressions or empty looks. So we think that concepts must be supported with material for quality in science education.

\subsection{Sentence of problem}

In this study, a model has been developed to make it easier to describe "the real and the apparent motions of the Sun-Earth-Moon system and the results of these motions", a topic within the scope of basic astronomy.

\subsection{Purpose of study}

The purpose of this study is to develop a Sun-Earth-Moon model which will be used in describing "the real and the apparent motions of the Sun-Earth-Moon system and the results of these motions", a fundamental topic in astronomy hardly understood correctly because it requires three-dimensional thinking and imagination. In this context;

- Students have difficulties in understanding fundamental concepts in astronomy such as formation of the seasons, phases of the Moon, lunar and solar eclipses which require three-dimensional thinking to be understood correctly. So, in order to teach difficultabstract concepts,

- In order to resolve shortage of material and tools related to the topic,

- In order to increase retention in learning by addressing multiple senses of students,

- Recording a concept visually and verbally in memory increase the ability to remember the image of that concept when the verbal counterpart of it is encountered, or vice versa. Therefore, in order to make it easier for students recall what they learn,

- In order to make students perceive tools, objects and events which they cannot perceive with the help of their five senses,

- In order to make students perceive tools, objects and events which they cannot reach or bring to the classroom setting,

- In order to draw attention and arouse curiosity for the topic,

a model has been needed to be developed.

\section{Extension activities}

According to our literature search, following serious studies related to our model have been published so far.

Ayres, Center, Bass, Carter and Bennett [1] developed "Colorado Scale Model Solar System". This model has been minimized in terms of distance and size. The Colorado Scale Model Solar System shows both planetary sizes and distances in our solar system at 1 tenbillionth of its actual size. The Sun and the inner planets have been placed in front of the Fiske Planetarium. This model is important because it is the only true scale model solar system in the world. According to this model, the Sun is roughly the size of a grapefruit (14 $\mathrm{cm}$ diameter), while the planets range in size from dust-speck-size Pluto to marble-size 
Jupiter. Earth is about the size of a pinhead (1,3 mm diameter). The inner planets (Mercury, Venus, Earth, and Mars) are all located within a couple dozen steps of the Sun, with the Earth located 15 meters from the Sun. The outer planets (Jupiter, Saturn, Uranus, Neptune, Pluto) are spread much farther apart with Pluto located about 600 meters from the Sun. Walking through the full length of the model would take about ten minutes, not including stops.

Toilet Paper Solar System: In this activity, students create a model of the solar system scaled to the distances between planets. Using sheets of toilet paper, students calculate the spacing between planets [13].

Planets Scaled to Size or Distance: By entering different measurements (metric or nonmetric) in a computer, models of solar system with different scales have been produced by Hipschman [7]. Students have the chance to make comparisons between the Sun and all the planets in terms of sizes and distances with this model [7].

The Voyage, a scaled model solar system, was opened in 2001 in Washington. Voyage depicts the Sun, the planets, and the distances between them all on the same scale of 1 to 10 billion. The Voyage exhibit was developed by the Challenger Center for Space Science Education, the Smithsonian Institution, and NASA [11]. One of the major goals of the Voyage project is to make people experience a walk through in a scaled solar system model.

The study by Diakidoy and Kendeou [3] has used the following materials to explain topics such as the shape of the Earth and the day-night cycle:

- a $64 \mathrm{~cm}$ by $40 \mathrm{~cm}$ map of Australia;

- a magnetic blue sphere, $15 \mathrm{~cm}$ in diameter;

- a magnetic toy ship, $7 \mathrm{~cm}$ long and $6,5 \mathrm{~cm}$ tall;

- a blue inflatable balloon, $125 \mathrm{~cm}$ in diameter;

- six globes;

- six flashlights.

The lesson begins with a presentation of the map of Australia and the students discuss what they know about this country. Subsequently, the location of Australia on the balloon is established, and it is argued how it is possible for people and objects to remain on Australian soil without falling off into space. The magnetic sphere and the magnetic toy ship are used to demonstrate the fact that the Earth behaves like a large magnet attracting everything to its surface. To observe that the Earth is a sphere, students move the toy ship along the surface of the balloon first, and then the surface of classroom table. Finally students hold flashlight to the rotated sphere and observe the day-night cycle.

In the study by Maclntyre, Stableford and Choudry [9], set up a mechanism by using off-the-shelf material to teach students the formation of the seasons and the change in the lengths of day and night.

In their study, Guy and Young [5] developed a Sun-Earth-Moon Model to teach solar and lunar eclipses by considering the distances and the sizes of the Sun, the Earth and the Moon. Features of this model has been described as follows:

- The Moon's diameter is about one quarter of Earth's diameter.

- The Moon is approximately 30 Earth diameters away from the Earth.

- There is a 5o angle between the orbital plane of the Moon around the Earth and that of the Earth around the Sun.

- Scale for size: Earth=1 cm, Moon=2-3 mm, Sun=1 m

- Scale for distance: Earth to Moon=30 cm, Sun to Earth=about $100 \mathrm{~m}$

- Materials: Plastic pins: 1-cm heads and 2-mm heads (for Earth and Moon), pencils, plastic rulers, overhead projector (for sunlight).

Students set up mechanisms by using the materials in accordance with the descriptions and the scales above and make observations. 


\section{Developing of the model}

The features of our model are as follows:

- The Sun rotates around its own axis.

- The Earth rotates around its own axis.

- The Moon rotates around its own axis.

- The Moon revolves in a circular orbit around the Earth and the angle between the orbital plane of the Moon around the Earth and that of the Earth around the Sun is 5,145o.

- The Moon and the Earth revolves in elliptical orbits around the Sun.

- Each of the three objects rotate around their axes and revolve around other bodies in the counter-clockwise direction.

Photos of the Sun, the Earth and the Moon and numerical data for our model are given in tables below.

Table 1. The real mean diameters and their corresponding values in our model

\begin{tabular}{ll}
\hline Real mean diameters $(\mathrm{km})$ & Mean diameters in our model $(\mathrm{cm})$ \\
\hline Earth's diameter: 12756,28 & Earth's diameter: 16 \\
Moon's diameter: 3474,13 & Moon's diameter: 4,3 \\
Sun's diameter: 1392000 & Sun's diameter: 30 \\
\hline
\end{tabular}

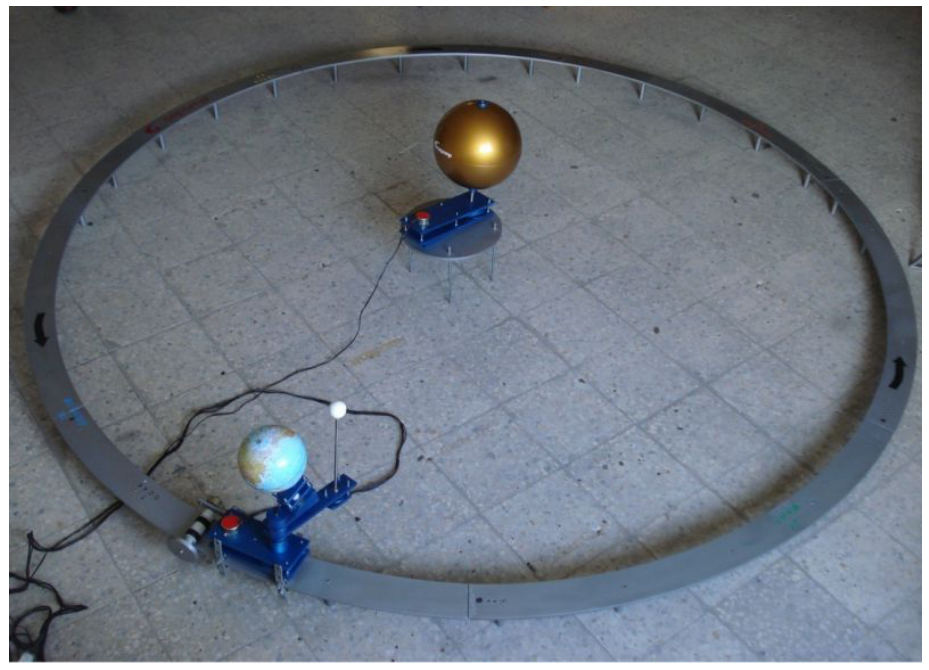

Fig. 1. Sun-Earth-Moon model

Table 2. The real tilts of axes and their corresponding values in our model

\begin{tabular}{ll}
\hline Real tilt of axis (degrees of angle) & Tilt of axis in the model (degree of angle) \\
\hline Earth's tilt of axis: 23,44 & Earth's tilt of axis: 23,44 \\
Moon's tilt of axis: 6,688 & Moon's tilt of axis: 6,688 \\
Sun's tilt of axis: 7,25 & Sun's tilt of axis: 7,25 \\
\hline
\end{tabular}




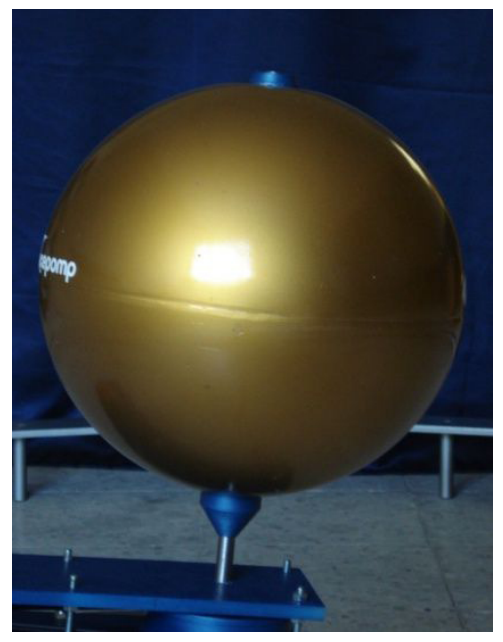

Fig. 2. Sun's tilt of axis.

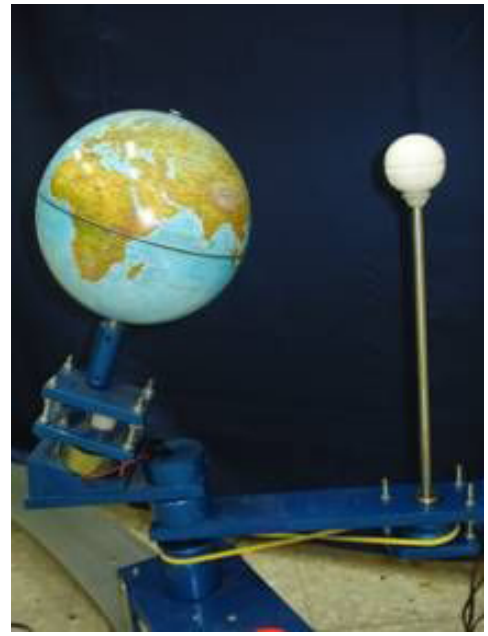

Fig. 3. Moon's and Earth's tilt of axis

Table 3. The real rotation periods and the rotation periods in our model

\begin{tabular}{ll}
\hline The real rotation periods & $\begin{array}{l}\text { The rotation periods in our } \\
\text { model }\end{array}$ \\
\hline $\begin{array}{l}\text { Rotation period of the Earth around its axis: 23,93 } \\
\text { hours (23 hr } 56 \mathrm{~min})\end{array}$ & $\begin{array}{l}\text { Rotation period of the Earth } \\
\text { around its axis: } 5,4 \mathrm{~s}\end{array}$ \\
$\begin{array}{l}\text { Rotation period of the Moon around its axis: } 27,32 \\
\text { days }\end{array}$ & $\begin{array}{l}\text { Rotation period of the Moon } \\
\text { around its axis: } 123 \mathrm{~s}\end{array}$ \\
$\begin{array}{l}\text { Rotation Period of the Sun around its axis: } 25,5 \\
\text { Rays }\end{array}$ & $\begin{array}{l}\text { Rotation period of the Sun } \\
\text { around its axis: } 107 \mathrm{~s} .\end{array}$ \\
\hline
\end{tabular}


Table 4. The real mean distances and the mean distances in our model

\begin{tabular}{ll}
\hline The Real Mean Distances (km) & The Mean Distances in our Model (cm) \\
\hline $\begin{array}{l}\text { The Earth's mean distance to the Sun: } 149597887 \\
\text { Aphelion distance (the Earth's farthest distance to }\end{array}$ & $\begin{array}{l}\text { The Earth's mean distance to the Sun: } 149,5 \\
\text { Aphelion distance (the Earth's farthest distance to } \\
\text { the Sun) on the Earth's orbit :152,1 }\end{array}$ \\
$\begin{array}{l}\text { Perihelion distance (the Earth's closest distance to } \\
\text { the Sun) on the Earth's orbit : } 147098074\end{array}$ & $\begin{array}{l}\text { Perihelion distance (the Earth's closest distance to } \\
\text { the Sun) on the Earth's orbit : } 147,1\end{array}$ \\
$\begin{array}{l}\text { The Moon's mean distance to the Earth: } 384399 \\
\text { Apogee distance (the Moon's farthest distance to the }\end{array}$ & $\begin{array}{l}\text { The Moon's mean distance to the Earth: } 19,2 \\
\text { Earth) on the Moon's orbit: } 405696\end{array}$ \\
$\begin{array}{l}\text { Perigee distance (the Moon's closest distance to the and perigee distances are mean distances. } \\
\text { Earth) on the Moon's orbit: } 363104\end{array}$ & $\begin{array}{l}\text { Because the Moon revolves around The Earth in a } \\
\text { circular orbit in our model (19,2 cm). }\end{array}$ \\
\hline
\end{tabular}

Note: Distances are from one centre to another.

Table 5. The real tilts of orbits and the tilts in our model

\begin{tabular}{ll} 
The real tilts of the orbits (degrees of angle) & $\begin{array}{l}\text { The Tilts of the Orbits in our Model } \\
\text { (degrees of angle) }\end{array}$ \\
\hline Tilt of the Earth's orbit: 0 & Tilt of the Earth's orbit: 0 \\
Tilt of the Moon's orbit: 5,145 & Tilt of the Moon's orbit: 5,145 \\
\hline
\end{tabular}

Table 6. The real revolution periods and the revolution periods in our model

\begin{tabular}{ll}
\hline The Real Revolution Periods (days) & $\begin{array}{l}\text { The Revolution Periods in our Model } \\
\text { (seconds) }\end{array}$ \\
\hline Revolution Period of the Earth around the & $\begin{array}{l}\text { Revolution Period of the Earth around } \\
\text { the Sun: 2033 }\end{array}$ \\
$\begin{array}{l}\text { Sun: } 365,256 \\
\text { Revolution Period of the Moon around the } \\
\text { Earth : 27,32 }\end{array}$ & $\begin{array}{l}\text { Revolution Period of the Moon } \\
\text { around the Earth: } 123\end{array}$ \\
\hline
\end{tabular}

\subsection{Limitations of our model}

The speed of the Earth's around the Sun on its elliptical orbit is kept constant. That is, the speed doesn't increase when the Earth approaches the Sun and doesn't decrease when it moves away.

The Earth's axis is not fixed. So by moving the Earth's axis from its head, its position is set manually, when the Earth revolves in its orbit.

When the Moon's diameter is accepted to be 4,3 cm in this model, the Sun's diameter is about $16 \mathrm{~m}$, the Earth's distance to the Sun is about 1,7 km and the Moon's distance to the Earth is about 4,42 m. Because of the lack of space in a laboratory environment, a true scale cannot be reached. Hence, the diameter of the Moon hasn't been accepted as a reference for the scale, but a new scale has been applied for these distances.

Although the Moon revolves around the Earth in an elliptical orbit, it is assumed to revolve in a circular one in our model.

The diameters of the Earth and the Moon have been scaled proportionally but the diameter of the Sun has been kept outside of this scale. When scaled, the Sun becomes a sphere with a distance of $1700 \mathrm{~m}$ to the Earth and a diameter of $16 \mathrm{~m}$. Therefore, it is not possible to apply the same scale to all of the bodies in this model. In addition, the Earth completes its one rotation around its axis in 5,4 seconds. When scaled, the Moon's rotational and orbital periods must be 147,5 seconds, the Sun's rotational period must be 
137,7 seconds and the Earth's orbital period must be 1972,3 seconds (32,87 minutes). Although these values have not been exactly achieved, they have been approached.

\section{Results}

Only the sizes of the Sun and the planets and the distances from each other have been taken into account, but the tilts of the axes, the tilts of the orbits, rotational and orbital motions have been ignored in the solar system models by Ayres et al [1], Slater [13] and Hipschman [7]. However, in the system developed by Ayres et al [1], many planets are too small because the Sun's diameter is $14 \mathrm{~cm}$. Therefore, we think that these planets cannot be distinguished from each other which will prevent the retention. Diakidoy and Kendeou [3] used different materials to replace the Earth and the Sun but the tilts of the axes, rotational and orbital motions have been ignored to be able to explain topics such as the shape of the Earth and the day-night cycle. Guy and Young [5], took into account only the distances and the sizes in their study. They gave the tilt of the Moon's orbit, and built independent mechanisms to illustrate lunar and solar eclipses but they ignore the tilts of the rotational and orbital axes, and motions in their solar system model. In this study, the tilts of the axes of the Sun, the Earth and the Moon, and rotational and orbital motions have been ignored. The Sun-Earth-Moon model which is developed by us is more complicated and detailed than the previous models in terms of explaining single setting topics such as eclipses, seasons, night-day cycle and moon phases, and having many features such as axial and orbital inclinations and motions at the same time. As a result of a literature review, this study proved to be superior to the other models in terms of its features. Thus our Sun-EarthMoon model stand out as the most detailed model in the field despite its mentioned limitations.

\section{Acknowledgment}

This study was supported by İnönü University, Department of Scientific Research Projects.

\section{References}

1. Ayres, T., Center, K., Bass, R., Carter, M., \& Bennett, J. The Colorado scale-model solar system. Physics Teacher, 29(6), 371-374, (1991).

2. Balkan, A. The Impact on Attitude, Success and Retention Toward Lesson of Using the Map at Social Lesson of Primary School Seventh Grade. Unpublished Master Thesis, University of Sakarya, Institute of Social Sciences, Sakarya-Turkey, (2007).

3. Diakidoy, I.-A.N., \& Kendeou, P. Facilitating conceptual change in astronomy: A comparison of the effectiveness of two instructional approaches. Learning and Instruction, 11, 1-20, (2001).

4. Gözmen, E. The Impact of Models in Teaching of Meiosis That Teach at High School First Biology Lesson. Unpublished Master Thesis, University of Selçuk, Institute of Natural Sciences, Konya-Turkey, (2008).

5. Guy, M., \& Young, T. Creating eclipses: Using scale models to explore how eclipses happen. Science Activities, 47, 75-82, (2010).

6. Güneş, M. H., \& Çelikler, D. The investigation of effects of modeling and computer assisted instruction on academic achievement. International Journal of Educational Researchers (IJER), 1(2), 22-28, (2010).

7. Hipschman, R., \& Exploratorium. Make a scale model of the solar system and learn the real definition of "space". Accessed 12 July 2012, http://www.exploratorium.edu/ronh/solarsystem, (1997). 
8. Koçak, E. The Impact on Student Success of Teaching Based Model of Topics Such as "Digestion and Responsible Structures", "Excretion and Responsible Structures" and "We Know A Flowering Plant" in the Primary Education Fifth Grade Students. Unpublished Master Thesis, University of Atatürk, Institute of Natural Sciences, Erzurum-Turkey, (2006).

9. Maclntyre, B., Stableford, J., \& Choudry, H. Teaching for conceptual understanding in astronomy: Using an investigating with models approach. Investigating, 18(1), 6-8, (2002).

10. Minasl1, E. The Impact on Success, Concept Learning and Remembering of Using Simulation and Model in Teaching of Topics Such as Structure and Properties of Matter in the Science Lesson. Unpublished Master Thesis, University of Marmara, Institute of Education Sciences, İstanbul-Turkey, (2009).

11. NASA, Challenger Center for Space Science Education and the Smithsonian Institution. Voyage Scale Model Solar System. Accessed 12 July 2012, http://www.jeffreybennett.com/co scale.html, (2001).

12. Sarıkaya, R., Selvi, M., \& Doğan Bora, N. The importance of using the model in teaching of mitosis and meiosis topics. Kastamonu Educational Journal, 12(1), 85-88, (2004).

13. Slater, T. The toilet paper solar system. Accessed 12 July 2012, http://solar.physics.montana.edu/tslater/ plunger/tissue.html, (1997).

14. Zeynelgiller, O. The Impact on Student Success of Using the Model in Chemistry Topics of Science Lesson of the Second Primary Education. Unpublished Master Thesis, University of Celal Bayar, Institute of Natural Sciences, Manisa-Turkey, (2006). 